\begin{tabular}{|c|c|c|}
\hline 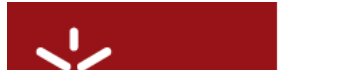 & CMAT & 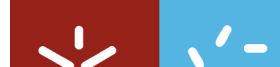 \\
\hline - Reprositónill & Centro de Matemática da Universidade do Minho & -0 \\
\hline \multirow[t]{2}{*}{ Universidade do Minho } & Campus de Gualtar $4710-057$ Braga Portugal & $\begin{array}{l}\text { Universidade do Minho } \\
\text { Escola de Ciências }\end{array}$ \\
\hline & www.cmat.uminho.pt & Centro de Matemática \\
\hline
\end{tabular}

\title{
Stationary quasivariational inequalities with gradient constraint and nonhomogeneous boundary conditions
}

\author{
Assis Azevedo Fernando Miranda Lisa Santos \\ CMAT and DMA, Universidade do Minho, Portugal
}

\section{Information}

Keywords:

Quasivariational inequality

Gradient constraint

Nonhomogeneous boundary conditions

Original publication:

C. Bernardin and P. Gonçalves (eds.), From Particle Systems to Partial Differential Equations, Springer Proceedings in Mathematics \& Statistics 75 (2014), 95-112

DOI: 10.1007/978-3-642-54271-8_2

www.springer.com

\begin{abstract}
We study existence of solution of stationary quasivariational inequalities with gradient constraint and nonhomogeneous boundary condition of Neumann or Dirichlet type. Through two different approaches, one making use of a fixed point theorem and the other using a process of regularization and penalization, we obtain different sufficient conditions for the existence of solution.
\end{abstract}

\section{Introduction and main results}

If we want to solve the well known problem of finding $u \in H_{0}^{1}(\Omega)$ such that

$$
\min \{-\Delta u-f, u-\psi\}=0 \quad \text { a.e. in } \Omega \text {, }
$$

for a given $\psi$, the easiest approach is to solve the variational inequality: to find $u \in K_{\psi}=\left\{v \in H_{0}^{1}(\Omega): v \geq\right.$ $\psi$ a.e. in $\Omega\}$ such that

$$
\int_{\Omega} \nabla u \cdot \nabla(v-u) \geq \int_{\Omega} f(v-u), \quad \forall v \in K_{\psi} .
$$

Existence of solution for stationary variational inequalities like the considered above is immediate (see [6]). Quasivariational inequalities are similar, but implicit, problems where the convex set depends on the solution. For instance, we consider the problem (1), with $K_{\psi}$ substituted by $K_{F(u)}$, for a given function $F \in \mathcal{C}(\mathbb{R})$. The proof of existence of solution is no more a trivial problem and different approaches can be used, such as a fixed point argument or approximation of the quasivariational inequality by a family of penalized equations, for which existence is known, using a priori estimates to pass to the limit.

Here we are interested in variational and quasivariational inequalities with gradient constraint, whose convex sets are of the following type:

$$
\mathbb{K}_{\varphi}=\left\{v \in W^{1, p}(\Omega):|\nabla v| \leq \varphi, \text { a.e. in } \Omega\right\}
$$


or

$$
\mathcal{K}_{\varphi}=\left\{v \in W^{1, p}(\Omega):|\nabla v| \leq \varphi, \text { a.e. in } \Omega, v_{\mid \partial \Omega}=g\right\},
$$

for $\varphi \geq 0$ in the variational case and $\varphi=F(u)$, in the quasivariational case, where $F \in \mathcal{C}(\mathbb{R})$ and $g \in \mathscr{C}(\partial \Omega)$.

The first model of this type was the elastoplastic torsion problem, a stationary variational inequality with gradient constraint 1 and homogeneous Dirichlet boundary condition ([18], [3] or [4]). Sand piles and river networks ([13] or [15]) or electromagnetic problems ([14], [17], [2], [10] or [11]) can be modeled by variational or quasivariational inequalities with gradient or curl constraint. As far as the authors know, the first work in quasivariational inequalities with gradient constraint and nonhomogeneous boundary condition is [1]. This work generalizes the existence results for quasivariational inequalities presented in that paper, improving the growth condition imposed on $F$ (details will be given later). We also present another situation where no growth condition is imposed on $F$, assuming that the operator considered is $\boldsymbol{a}(x, \boldsymbol{u})=a(x)|\boldsymbol{u}|^{p-2} \boldsymbol{u}$ and assuming a little more on the regularity of the data. We notice that, assuming nonhomegeneous conditions on the boundary introduces additional difficulties when seeking for solutions of quasivariational inequalities. The proof of existence of solution may be done either using a fixed point theorem or by approximating the quasivariational inequality by a family of equations. In both cases, given a function in a certain convex set (depending on the constraint of the gradient and on the boundary condition), we need to find out a function in another convex set and estimate their distance. This procedure, not easy even when null boundary conditions are considered in both convex sets, becomes harder when the boundary conditions change, situation scarcely considered in the literature.

In this paper, we consider $\Omega$ a bounded open subset of $\mathbb{R}^{N}$ with smooth boundary. Given $1<p<\infty$, let $\boldsymbol{a}: \Omega \times \mathbb{R}^{N} \longrightarrow \mathbb{R}^{N}$ be a Carathéodory function satisfying the structural conditions (4a), (4b) and (4c) or $\left(4 c^{\prime}\right)$

$$
\begin{aligned}
\boldsymbol{a}(x, \boldsymbol{u}) \cdot \boldsymbol{u} & \geq a_{*}|\boldsymbol{u}|^{p}, \\
|\boldsymbol{a}(x, \boldsymbol{u})| & \leq a^{*}|\boldsymbol{u}|^{p-1}, \\
(\boldsymbol{a}(x, \boldsymbol{u})-\boldsymbol{a}(x, \boldsymbol{v})) \cdot(\boldsymbol{u}-\boldsymbol{v}) & >0, \text { if } \boldsymbol{u} \neq \boldsymbol{v}, \\
(\boldsymbol{a}(x, \boldsymbol{u})-\boldsymbol{a}(x, \boldsymbol{v})) \cdot(\boldsymbol{u}-\boldsymbol{v}) & \geq \begin{cases}a_{*}|\boldsymbol{u}-\boldsymbol{v}|^{p} & \text { if } p \geq 2, \\
a_{*}(|\boldsymbol{u}|+|\boldsymbol{v}|)^{p-2}|\boldsymbol{u}-\boldsymbol{v}|^{2} & \text { if } p<2,\end{cases}
\end{aligned}
$$

for given constants $0<a_{*}<a^{*}$, for all $\boldsymbol{u}, \boldsymbol{v} \in \mathbb{R}^{N}$ and a.e. $x \in \Omega$.

Let $q$ be the critical Sobolev exponent of $p$, if $p \neq N$, i.e.,

$$
q=\frac{N p}{N-p} \text { if } 1<p<N, \quad q=\infty \text { if } p>N,
$$

and $q>1$, if $p=N$. Observe that, given $v \in W^{1, p}(\Omega)$, we have the following inequality

$$
\|v\|_{L^{q}(\Omega)} \leq C_{q}\|v\|_{W^{1, p}(\Omega)},
$$

being $C_{q}>0$.

Let $r$ be the critical Sobolev exponent of $p$ for the trace embedding, if $p \neq N$, i.e.,

$$
r=\frac{(N-1) p}{N-p} \text { if } 1<p<N, \quad r=\infty \text { if } p>N,
$$

and $r>1$, if $p=N$. Then, given $v \in W^{1, p}(\Omega)$, there exists $C_{r}>0$ such that

$$
\|v\|_{L^{r}(\partial \Omega)} \leq C_{r}\|v\|_{W^{1, p}(\Omega)} .
$$

Given

$$
F \in \mathscr{C}\left(\mathbb{R} ; \mathbb{R}^{+}\right), f \in L^{q^{\prime}}(\Omega), g \in L^{r^{\prime}}(\partial \Omega), c \in L^{\infty}(\Omega), c \geq c_{*},
$$

where $c_{*}$ is a nonnegative constant, consider the following quasivariational inequality with Neumann type boundary condition: to find $u \in \mathbb{K}_{F(u)}$ such that

$$
\int_{\Omega} \boldsymbol{a}(x, \nabla u) \cdot \nabla(v-u)+\int_{\Omega} c|u|^{p-2} u(v-u) \geq \int_{\Omega} f(v-u)+\int_{\partial \Omega} g(v-u), \quad \forall v \in \mathbb{K}_{F(u)},
$$


where $\mathbb{K}_{F(u)}$ is defined in (2).

The following two theorems give sufficient conditions for existence of solution of the above quasivariational inequality.

Theorem 1.1 Assume (4a), (4b), (4c') and (7), with $c_{*}>0$. If $p \leq N$ suppose, in addition, that there exist positive constants $c_{0}$ and $c_{1}$ such that

$$
F(s) \leq c_{0}+c_{1}|s|^{\alpha}, \quad \forall s \in \mathbb{R},
$$

being $\alpha \geq 0$ if $p=N$ and $0 \leq \alpha<\frac{N}{N-p}$ if $p<N$.

Then the quasivariational inequality (8) has a solution.

Remark 1.2 We point out that the condition $0 \leq \alpha<\frac{p}{N-p}$ when $p<N$ assumed in [1] is here improved to $0 \leq \alpha<\frac{N}{N-p}$.

The following theorem states existence of solution for problem (8) with homogeneous Neumann boundary condition, imposing no growth condition on $F$ but assuming the strict positivity of $F$, the boundedness of $f$ and a restriction on the operator $\boldsymbol{a}$.

Theorem 1.3 Assume that $\boldsymbol{a}(x, \boldsymbol{u})=a(x)|\boldsymbol{u}|^{p-2} \boldsymbol{u}$ with $0<a_{*} \leq a \leq a^{*}$. Assume, in addition, that $f \in L^{\infty}(\Omega), g \equiv 0, c \in L^{\infty}(\Omega)$, with $c \geq c_{*}$, and $F \in \mathscr{C}\left(\mathbb{R} ; \mathbb{R}^{+}\right)$, with $F \geq F_{*}$, where $c_{*}$ and $F_{*}$ are positive constants.

Then the quasivariational inequality (8) has a solution.

Consider the quasivariational inequality with Dirichlet type boundary condition: to find $u \in \mathcal{K}_{F(u)}$ such that

$$
\int_{\Omega} \boldsymbol{a}(x, \nabla u) \cdot \nabla(v-u)+\int_{\Omega} c|u|^{p-2} u(v-u) \geq \int_{\Omega} f(v-u), \quad \forall v \in \mathcal{K}_{F(u)},
$$

where $\mathcal{K}_{F(u)}$ is defined in (3).

We present two theorems which give sufficient conditions for the existence of solution of the above quasivariational inequality.

Theorem 1.4 Consider the assumptions of Theorem 1.1, with $c_{*} \geq 0$ and $F \geq F_{*}>0$, where $c_{*}$ and $F_{*}$ are constants. Assume, in addition, that there exists $k \in[0,1)$ such that

$$
|g(x)-g(y)| \leq k F_{*} \bar{d}(x, y) \quad \text { for } x, y \in \partial \Omega,
$$

where $\bar{d}$ is the geodesic distance in $\Omega$.

Then the quasivariational inequality (9) has a solution.

We observe that the above theorem generalizes a result of [7], where Dirichlet homogeneous boundary condition was considered as well as a more restrictive growth assumption on $F$, for $1<p \leq N$.

Theorem 1.5 Assume that $\boldsymbol{a}(x, \boldsymbol{u})=a(x)|\boldsymbol{u}|^{p-2} \boldsymbol{u}$ with $0<a_{*} \leq a \leq a^{*}$. Assume in addition, that (10) is verified for some $k<\frac{a_{*}}{a^{*}}, f \in L^{\infty}(\Omega), c \in L^{\infty}(\Omega)$, with $c \geq c_{*}, \bar{F} \in \mathscr{C}\left(\mathbb{R} ; \mathbb{R}^{+}\right)$, with $F \geq F_{*}$, where $c_{*}, F_{*}$ are constants, $c_{*} \geq 0$ and $F_{*}>0$.

Then the quasivariational inequality (9) has a solution.

\section{The case with Neumann boundary condition}

In this section we consider the quasivariational inequality with Neumann boundary condition. The proof of Theorem 1.1 uses a fixed point theorem and the proof of Theorem 1.3 is done by approximating the quasivariational inequality by a family of penalized and regularized equations.

Given $\varphi \in L^{\infty}(\Omega), \varphi \geq 0$, we consider the variational inequality: to find $u \in \mathbb{K}_{\varphi}$ such that

$$
\int_{\Omega} \boldsymbol{a}(x, \nabla u) \cdot \nabla(v-u)+\int_{\Omega} c|u|^{p-2} u(v-u) \geq \int_{\Omega} f(v-u)+\int_{\partial \Omega} g(v-u), \quad \forall v \in \mathbb{K}_{\varphi},
$$

where $\mathbb{K}_{\varphi}$ is defined in (2). In this section we assume (4a), (4b), (4c) and (7) with $c_{*}>0$. Under these assumptions, this problem has a unique solution (see [8, Theorem 8.2]). 
Proposition 2.1 Let $u$ be the solution of problem (11). Then

$$
\|u\|_{W^{1, p}(\Omega)} \leq M\left(\|f\|_{L^{q^{\prime}}(\Omega)}+\|g\|_{L^{r^{\prime}}(\partial \Omega)}\right)^{\frac{1}{p-1}} .
$$

where $M=\left(\frac{\max \left\{C_{q}, C_{r}\right\}}{\min \left\{a_{*}, c_{*}\right\}}\right)^{\frac{1}{p-1}}$, for $C_{q}$ and $C_{r}$ defined in (5) and in (6).

Proof Considering $v=0$ in the variational inequality (11) we obtain,

$$
\begin{aligned}
\int_{\Omega} \boldsymbol{a}(x, \nabla u) \cdot \nabla u+\int_{\Omega} c|u|^{p} & \leq \int_{\Omega} f u+\int_{\partial \Omega} g u \\
& \leq\|f\|_{L^{q^{\prime}}(\Omega)}\|u\|_{L^{q}(\Omega)}+\|g\|_{L^{r^{\prime}(\partial \Omega)}}\|u\|_{L^{r}(\partial \Omega)} \\
& \leq C\left(\|f\|_{L^{q^{\prime}(\Omega)}}+\|g\|_{L^{r^{\prime}(\partial \Omega)}}\right)\|u\|_{W^{1, p}(\Omega)},
\end{aligned}
$$

where $C=\max \left\{C_{q}, C_{r}\right\}$. But, as

$$
\min \left\{a_{*}, c_{*}\right\}\|u\|_{W^{1, p}(\Omega)}^{p} \leq a_{*}\|\nabla u\|_{L^{p}(\Omega)}^{p}+c_{*}\|u\|_{L^{p}(\Omega)}^{p} \leq \int_{\Omega} \boldsymbol{a}(x, \nabla u) \cdot \nabla(u)+\int_{\Omega} c|u|^{p},
$$

the conclusion follows.

We present now a continuous dependence result on the gradient constraints that will be necessary to apply later a fixed point theorem. A more general result can be found in [1], where the dependence on $f$ and $g$ is also considered.

Proposition 2.2 For $\varphi, \psi \in L^{\infty}(\Omega)$ with a positive lower bound $\eta$ and a verifying (4a), (4b) and (4c'), the solutions $u_{\varphi}$ and $u_{\psi}$ of problem (11) satisfy

$$
\left\|u_{\varphi}-u_{\psi}\right\|_{W^{1, p}(\Omega)}^{\max \{p, 2\}} \leq C\|\varphi-\psi\|_{L^{\infty}(\Omega)},
$$

where $C=C(\eta)$ is a positive constant.

Proof Letting $A(u, v)=\int_{\Omega} \boldsymbol{a}(x, \nabla u) \cdot \nabla v+\int_{\Omega} c|u|^{p-2} u v$, then, for $\lambda \in \mathbb{R}$,

$$
A(u, u-v)-A(v, u-v)=A(u, u-\lambda v)+A(v, v-\lambda u)+(\lambda-1)[A(u, v)+A(v, u)] .
$$

Recall that $\eta$ is a positive lower bound of $\varphi$ and $\psi$ and set $\lambda=\frac{\eta}{\eta+\|\varphi-\psi\|_{\infty}}$. Then, as $\lambda u_{\psi} \in K_{\varphi}$ and $\lambda u_{\varphi} \in K_{\psi}$, using $\lambda u_{\varphi}$ as test function in (11) with convex set $\mathbb{K}_{\psi}$ and $\lambda u_{\psi}$ as test function in (11) with convex set $\mathbb{K}_{\varphi}$ we have,

$$
\begin{aligned}
A\left(u_{\varphi}, u_{\varphi}-\lambda u_{\psi}\right)+A\left(u_{\psi}, u_{\psi}-\lambda u_{\varphi}\right) \leq(1-\lambda)\left(\int_{\Omega} f\left(u_{\varphi}+u_{\psi}\right)+\int_{\partial \Omega} g\left(u_{\varphi}+u_{\psi}\right)\right) \\
\leq(1-\lambda) C\left(\|f\|_{L^{q^{\prime}}(\Omega)}+\|g\|_{L^{r^{\prime}}(\partial \Omega)}\right)\left(\left\|u_{\varphi}\right\|_{W^{1, p}(\Omega)}+\left\|u_{\psi}\right\|_{W^{1, p}(\Omega)}\right) \leq \frac{D}{\eta}\|\varphi-\psi\|_{L^{\infty}(\Omega)}
\end{aligned}
$$

where $C=\max \left\{C_{q}, C_{r}\right\}$ and $D=D\left(\|f\|_{L^{q^{\prime}}(\Omega)},\|g\|_{L^{r^{\prime}}(\partial \Omega)}\right)$ is a positive constant. The last inequality is true by Proposition 2.1 and because

$$
1-\lambda=\frac{\|\varphi-\psi\|_{L^{\infty}(\Omega)}}{\eta+\|\varphi-\psi\|_{L^{\infty}(\Omega)}} \leq \frac{\|\varphi-\psi\|_{L^{\infty}(\Omega)}}{\eta} .
$$

On the other hand, recalling the constant $M$ defined in Proposition 2.1,

$$
\begin{aligned}
\left|A\left(u_{\varphi}, u_{\psi}\right)\right| & \leq a^{*} \int_{\Omega}\left|\nabla u_{\varphi}\right|^{p-1}\left|\nabla u_{\psi}\right|+\|c\|_{L^{\infty}(\Omega)} \int_{\Omega}\left|u_{\varphi}\right|^{p-1}\left|u_{\psi}\right| \\
& \leq a^{*}\left\|\nabla u_{\varphi}\right\|_{L^{p}(\Omega)}^{p-1}\left\|\nabla u_{\psi}\right\|_{L^{p}(\Omega)}+\|c\|_{L^{\infty}(\Omega)}\left\|u_{\varphi}\right\|_{L^{p}(\Omega)}^{p-1}\left\|u_{\psi}\right\|_{L^{p}(\Omega)} \\
& \leq\left(a^{*}+\|c\|_{L^{\infty}(\Omega)}\right)\left\|u_{\varphi}\right\|_{W^{1, p}(\Omega)}^{p-1}\left\|u_{\psi}\right\|_{W^{1, p}(\Omega)} \\
& \leq\left(a^{*}+\|c\|_{L^{\infty}(\Omega)}\right) M^{p}\left(\|f\|_{L^{q^{\prime}}(\Omega)}+\|g\|_{L^{r^{\prime}(\partial \Omega)}}\right)^{p^{\prime}}
\end{aligned}
$$


and, analogously,

$$
\left|A\left(u_{\psi}, u_{\varphi}\right)\right| \leq\left(a^{*}+\|c\|_{L^{\infty}(\Omega)}\right) M^{p}\left(\|f\|_{L^{q^{\prime}}(\Omega)}+\|g\|_{L^{r^{\prime}}(\partial \Omega)}\right)^{p^{\prime}} .
$$

So, using (12), there exists $C=C\left(\|f\|_{L^{q^{\prime}}(\Omega)},\|g\|_{L^{r^{\prime}}(\partial \Omega)}, \eta\right)>0$ such that

$$
A\left(u_{\varphi}, u_{\varphi}-u_{\psi}\right)-A\left(u_{\psi}, u_{\varphi}-u_{\psi}\right) \leq C\|\varphi-\psi\|_{L^{\infty}(\Omega)} .
$$

On the other hand, by $\left(4 c^{\prime}\right)$

$$
A\left(u_{\varphi}, u_{\varphi}-u_{\psi}\right)-A\left(u_{\psi}, u_{\varphi}-u_{\psi}\right) \geq \min \left\{a_{*}, c_{*}\right\}\left\|u_{\varphi}-u_{\psi}\right\|_{W^{1, p}(\Omega)}^{p} \quad \text { if } p \geq 2 .
$$

Using the reverse Hölder inequality in the case $p<2$, we get

$$
\begin{aligned}
A\left(u_{\varphi}, u_{\varphi}-u_{\psi}\right) & -A\left(u_{\psi}, u_{\varphi}-u_{\psi}\right) \\
& \geq a_{*}\left\|\left|\nabla u_{\varphi}\right|+\left|\nabla u_{\psi}\right|\right\|_{L^{p}(\Omega)}^{p-2}\left\|\nabla u_{\varphi}-\nabla u_{\psi}\right\|_{L^{p}(\Omega)}^{2}+c_{*}\left\|\left|u_{\varphi}\right|+\left|u_{\psi}\right|\right\|_{L^{p}(\Omega)}^{p-2}\left\|u_{\varphi}-u_{\psi}\right\|_{L^{p}(\Omega)}^{2}
\end{aligned}
$$

and then, by Proposition 2.1, the conclusion follows also in this case.

The following proposition will be used in the proof of Theorem 1.1.

Proposition 2.3 Let $N \in \mathbb{N}, p>1, s>N, \frac{N}{N-1}<\alpha$ and $\alpha<\frac{N}{N-p}$ if $p<N$. Consider the sequence $\left(s_{n}\right)_{n}$ defined by

$$
s_{1}=s \quad \text { and } \quad s_{n+1}=\frac{\alpha N s_{n}}{N+\alpha s_{n}} .
$$

Then there exists $n \in \mathbb{N}$ such that $1<s_{n} \leq p$.

Proof Using the inequality $\frac{N}{N-1}<\alpha$ it is easy to prove, by induction, that $s_{n}>1$ for all $n \in \mathbb{N}$. On the other hand $\left(s_{n}\right)_{n}$ is a decreasing sequence, because $s_{2}<s_{1}$ and, for $n>2$,

$$
s_{n+1}<s_{n} \Leftrightarrow \frac{\alpha s_{n} N}{N+\alpha s_{n}}<\frac{\alpha s_{n-1} N}{N+\alpha s_{n-1}} \Leftrightarrow \frac{s_{n}}{N+\alpha s_{n}}<\frac{s_{n-1}}{N+\alpha s_{n-1}} \Leftrightarrow s_{n}<s_{n-1} .
$$

So $\left(s_{n}\right)_{n}$ is convergent. Using the equality $s_{n+1}=\frac{\alpha s_{n} N}{N+\alpha s_{n}}$, we see that the limit is $N\left(1-\frac{1}{\alpha}\right)$. To conclude we just need to observe that $N\left(1-\frac{1}{\alpha}\right)<p$. This is true because if $p<N, \alpha<\frac{N}{N-p}$.

We are now able to prove our first result.

Proof of Theorem 1.1 Consider a sequence $\left(p_{n}\right)_{n}$ such that $p_{1}=p$ and, for $i \geq 1, p_{i}$ is a critical Sobolev exponent of $p_{i-1}$. Let $s$ be the first element of this sequence greater than $N$. Applying repeatedly the Sobolev type inequality (5) one has

$$
\exists C>0 \forall u \in W^{1, s}(\Omega) \quad\|u\|_{W^{1, s}(\Omega)} \leq C\left(\|u\|_{L^{p}(\Omega)}+\|\nabla u\|_{L^{s}(\Omega)}\right) .
$$

Observe that, if $\varphi \in \mathscr{C}(\bar{\Omega})$ and $u \in \mathcal{K}_{F(\varphi)}$ then $u \in W^{1, s}(\Omega)$, as $\nabla u \in L^{\infty}(\Omega)$. In particular, the operator $T: \mathscr{C}(\bar{\Omega}) \longrightarrow W^{1, s}(\Omega)$ such that $T(\varphi)=u_{\varphi}$, where $u_{\varphi}$ is the solution of problem (11) with $\mathcal{K}_{F(\varphi)}$ replacing $\mathcal{K}_{\varphi}$, is well-defined.

To prove that $T$ is continuous, consider $\varphi \in \mathscr{C}(\bar{\Omega})$ and let $\delta>0$ be such that $\|F(\psi)\|_{\mathscr{C}(\bar{\Omega})} \leq\|F(\varphi)\|_{\mathscr{C}(\bar{\Omega})}+$ 1 if $\|\varphi-\psi\|_{\mathscr{C}(\bar{\Omega})} \leq \delta$. For those $\psi$ we have,

$$
\begin{aligned}
\left|\nabla u_{\varphi}-\nabla u_{\psi}\right|^{s} & =\left|\nabla u_{\varphi}-\nabla u_{\psi}\right|^{s-p}\left|\nabla u_{\varphi}-\nabla u_{\psi}\right|^{p} \\
& \leq\left(\left|\nabla u_{\varphi}\right|+\left|\nabla u_{\psi}\right|\right)^{s-p}\left|\nabla u_{\varphi}-\nabla u_{\psi}\right|^{p} \leq(F(\varphi) \\
& +F(\psi))^{s-p}\left|\nabla u_{\varphi}-\nabla u_{\psi}\right|^{p} \\
& \leq\left(2\|F(\varphi)\|_{\mathscr{C}(\bar{\Omega})}+1\right)^{s-p}\left|\nabla u_{\varphi}-\nabla u_{\psi}\right|^{p}
\end{aligned}
$$

and then, using (13),

$$
\left\|u_{\varphi}-u_{\psi}\right\|_{W^{1, s}(\Omega)} \leq C\left(\left\|u_{\varphi}-u_{\psi}\right\|_{L^{p}(\Omega)}+\left(2\|F \circ \varphi\|_{\mathscr{C}(\bar{\Omega})}+1\right)^{\frac{s-p}{s}}\left\|\nabla\left(u_{\varphi}-u_{\psi}\right)\right\|_{L^{p}(\Omega)}^{\frac{p}{s}}\right) .
$$


Noticing that $F(\varphi)$ and $F(\psi)$ has a positive lower bound, as $\varphi, \psi \in \mathscr{C}(\bar{\Omega})$ and $F \in \mathscr{C}\left(\mathbb{R} ; \mathbb{R}^{+}\right)$, this last inequality together with the Proposition 2.2, proves that $T$ is continuous.

In order to apply a fixed point theorem we consider

$$
S=i \circ T: \mathscr{C}(\bar{\Omega}) \longrightarrow \mathscr{C}(\bar{\Omega}),
$$

where $i$ is the compact inclusion of $W^{1, s}(\Omega)$ in $\mathscr{C}(\bar{\Omega})$. If $p>N$ then $s=p$ and Proposition 2.1 shows that $T$ is bounded and so, as $s>N$, the image of $S$ is compact and the conclusion follows from the Schauder fixed point theorem.

If $p \leq N$ we use the Leray-Schauder fixed point theorem. As $i$ is compact we only need to prove the boundedness in $W^{1, s}(\Omega)$ of the set

$$
\mathcal{A}=\{\varphi \in \mathscr{C}(\bar{\Omega}): \varphi=\lambda S(\varphi) \text { for some } \lambda \in[0,1]\} .
$$

Notice that we can suppose that $\alpha>\frac{N}{N-1}$. Consider the sequence defined in Proposition 2.3 starting with $s$ and let $n$ be such that $1<s_{n} \leq p$.

If $\varphi \in \mathcal{A}$ we have

$$
\left|\nabla u_{\varphi}\right| \leq F(\varphi) \leq c_{0}+c_{1}|\varphi|^{\alpha}=c_{0}+c_{1} \lambda^{\alpha}\left|u_{\varphi}\right|^{\alpha}
$$

and then, for $i<n$, there exist $A, D>0$, such that

$$
\begin{aligned}
\left\|u_{\varphi}\right\|_{W^{1, s_{i-1}}(\Omega)} \leq A\left(\left\|u_{\varphi}\right\|_{L^{\alpha s_{i-1}}(\Omega)}+\left\|\nabla u_{\varphi}\right\|_{L^{s_{i-1}}(\Omega)}\right), \quad \text { as } \alpha>1 \\
\leq A\left(\left\|u_{\varphi}\right\|_{L^{\alpha s_{i-1}}(\Omega)}+c_{0}|\Omega|^{\frac{1}{s_{i-1}}}+c_{1} \lambda^{\alpha}\left\|u_{\varphi}\right\|_{L^{\alpha s_{i-1}}(\Omega)}^{\alpha}\right) \\
\leq A\left(D\left\|u_{\varphi}\right\|_{W^{1, s_{i}}(\Omega)}+c_{0}|\Omega|^{\frac{1}{s_{i-1}}}+c_{1} \lambda^{\alpha} D\left\|u_{\varphi}\right\|_{W^{1, s_{i}}(\Omega)}^{\alpha}\right) \\
\quad \text { as } \alpha s_{i-1} \text { is the critical Sobolev exponent of } s_{i} .
\end{aligned}
$$

By consequence, using Proposition 2.1 and since $s_{n} \leq p$, we obtain the boundedness of $\mathcal{A}$ in $W^{1, s}(\Omega)$. So $T$ has a fixed point and this fixed point solves the quasivariational inequality.

The proof of Theorem 1.3 will be done using a family of approximating problems, obtained by regularizing and penalizing the quasivariational inequality.

Given $0<\varepsilon<1$, consider the family of quasilinear elliptic problems

$$
\begin{gathered}
-\nabla \cdot\left(k_{\varepsilon}\left(\left|\nabla u^{\varepsilon}\right|^{p}-F_{\varepsilon}^{p}\left(u^{\varepsilon}\right)\right) a_{\varepsilon}(x)\left(\left|\nabla u^{\varepsilon}\right|^{2}+\varepsilon\right)^{\frac{p-2}{2}} \nabla u^{\varepsilon}+\varepsilon \nabla u^{\varepsilon}\right)+c\left|u^{\varepsilon}\right|^{p-2} u^{\varepsilon}=f^{\varepsilon} \quad \text { in } \Omega, \\
\left(k_{\varepsilon}\left(\left|\nabla u^{\varepsilon}\right|^{p}-F_{\varepsilon}^{p}\left(u^{\varepsilon}\right)\right) a_{\varepsilon}(x)\left(\left|\nabla u^{\varepsilon}\right|^{2}+\varepsilon\right)^{\frac{p-2}{2}} \nabla u^{\varepsilon}+\varepsilon \nabla u^{\varepsilon}\right) \cdot \boldsymbol{n}=0 \quad \text { on } \partial \Omega,
\end{gathered}
$$

where $a_{\varepsilon}, f^{\varepsilon}$ and $F_{\varepsilon}$ are approximations by convolution of $a, f$ and $F$, and $k_{\varepsilon}$ is a smooth nondecreasing function such that

$$
k_{\varepsilon}(s)= \begin{cases}1 & \text { if } s \leq 0, \\ e^{\frac{s}{\varepsilon}} & \text { if } \varepsilon \leq s .\end{cases}
$$

This problem has a unique solution $u^{\varepsilon} \in \mathscr{C}^{2, \alpha}(\Omega) \cap \mathscr{C}(\bar{\Omega})$, being the proof a simple adaptation of [5, Theorem 5.19] for the case with Neumann homogeneous boundary condition.

Before proving Theorem 1.3 we need the following auxiliary result.

Proposition 2.4 Let $u^{\varepsilon}$ be a solution of problem (14). Then there exist positive constants $C_{1}, C_{2}$ and $D_{q}$, independent of $\varepsilon$, such that

$$
\begin{gathered}
\left\|u^{\varepsilon}\right\|_{L^{\infty}(\Omega)} \leq C_{1}, \\
\left\|k_{\varepsilon}\left(\left|\nabla u^{\varepsilon}\right|^{p}-F^{p}\left(u^{\varepsilon}\right)\right)\right\|_{L^{1}(\Omega)} \leq C_{2}, \\
\forall 1<q<\infty \quad\left\|\nabla u_{\varepsilon}\right\|_{L^{q}(\Omega)} \leq D_{q} .
\end{gathered}
$$


Proof Denote, for simplicity, $w=\left|\nabla u^{\varepsilon}\right|^{p}-F_{\varepsilon}^{p}\left(u^{\varepsilon}\right)$. Consider $\gamma \in \mathbb{R}^{+}$, to be chosen later. Multiplying equation (14a) by $\left(u^{\varepsilon}-\gamma\right)^{+}$and integrating over $\Omega$, we get

$$
\begin{aligned}
\int_{\Omega} k_{\varepsilon}(w) a_{\varepsilon}(x)\left(\left|\nabla u^{\varepsilon}\right|^{2}+\varepsilon\right)^{\frac{p-2}{2}} & \nabla u^{\varepsilon} \cdot \nabla\left(u^{\varepsilon}-\gamma\right)^{+} \\
& +\varepsilon \int_{\Omega} \nabla u^{\varepsilon} \cdot \nabla\left(u^{\varepsilon}-\gamma\right)^{+}+\int_{\Omega} c\left|u^{\varepsilon}\right|^{p-2} u^{\varepsilon}\left(u^{\varepsilon}-\gamma\right)^{+}=\int_{\Omega} f^{\varepsilon}\left(u^{\varepsilon}-\gamma\right)^{+}
\end{aligned}
$$

and so

$$
\begin{aligned}
\int_{\Omega} k_{\varepsilon}(w) a_{\varepsilon}(x)\left(\left|\nabla\left(u^{\varepsilon}-\gamma\right)^{+}\right|^{2}+\varepsilon\right)^{\frac{p-2}{2}}\left|\nabla\left(u^{\varepsilon}-\gamma\right)^{+}\right|^{2} & \\
& +\varepsilon \int_{\Omega}\left|\nabla\left(u^{\varepsilon}-\gamma\right)^{+}\right|^{2}+\int_{\Omega} c\left|u^{\varepsilon}\right|^{p-1}\left(u^{\varepsilon}-\gamma\right)^{+} \leq\left\|f^{\varepsilon}\right\|_{L^{\infty}(\Omega)} \int_{\Omega}\left(u^{\varepsilon}-\gamma\right)^{+} .
\end{aligned}
$$

Observing that the two first terms of the above inequality are nonegative and choosing $\gamma>\left(\frac{\|f\|_{L^{\infty}(\Omega)}}{c_{*}}\right)^{\frac{1}{p-1}}$, we get

$$
\left(c_{*} \gamma^{p-1}-\left\|f^{\varepsilon}\right\|_{L^{\infty}(\Omega)}\right) \int_{\Omega}\left(u^{\varepsilon}-\gamma\right)^{+} \leq 0
$$

and so $\left(u^{\varepsilon}-\gamma\right)^{+} \equiv 0$. Proceeding similarly, we obtain $\left(u^{\varepsilon}+\gamma\right)^{-} \equiv 0$, concluding (16).

Multiply now equation (14a) by $u^{\varepsilon}$ and integrate in $\Omega$. Then

$$
\int_{\Omega} k_{\varepsilon}(w) a_{\varepsilon}(x)\left(\left|\nabla u^{\varepsilon}\right|^{2}+\varepsilon\right)^{\frac{p-2}{2}}\left|\nabla u^{\varepsilon}\right|^{2}+\varepsilon \int_{\Omega}\left|\nabla u^{\varepsilon}\right|^{2}+\int_{\Omega} c\left|u^{\varepsilon}\right|^{p}=\int_{\Omega} f^{\varepsilon} u^{\varepsilon} .
$$

Observe that

$$
\begin{aligned}
\int_{\Omega} k_{\varepsilon}(w) a_{\varepsilon}(x)\left(\left|\nabla u^{\varepsilon}\right|^{2}+\varepsilon\right)^{\frac{p-2}{2}}\left|\nabla u^{\varepsilon}\right|^{2}=\int_{\Omega} k_{\varepsilon}(w) a_{\varepsilon}(x)\left(\left|\nabla u^{\varepsilon}\right|^{2}+\varepsilon\right)^{\frac{p}{2}} & \\
& -\varepsilon \int_{\Omega} k_{\varepsilon}(w) a_{\varepsilon}(x)\left(\left|\nabla u^{\varepsilon}\right|^{2}+\varepsilon\right)^{\frac{p-2}{2}}
\end{aligned}
$$

and it can be easily seen that

$$
\varepsilon \int_{\Omega} k_{\varepsilon}(w) a_{\varepsilon}(x)\left(\left|\nabla u^{\varepsilon}\right|^{2}+\varepsilon\right)^{\frac{p-2}{2}} \leq \alpha_{\varepsilon} \int_{\Omega} k_{\varepsilon}(w)\left|\nabla u^{\varepsilon}\right|^{p}+\beta_{\varepsilon} \int_{\Omega} k_{\varepsilon}(w),
$$

where $\alpha_{\varepsilon} \underset{\varepsilon \rightarrow 0}{\longrightarrow} 0$ and $\beta_{\varepsilon} \underset{\varepsilon \rightarrow 0}{\longrightarrow} 0$.

Noticing that $k_{\varepsilon}(w) F_{*}^{p} \leq k_{\varepsilon}(w)\left|\nabla u^{\varepsilon}\right|^{p}+F_{\varepsilon}^{p}\left(u^{\varepsilon}\right)$ since $k_{\varepsilon}(w)=1$ if $w \leq 0$ and, when $w>0$, we have $\left|\nabla u^{\varepsilon}\right| \geq F_{\varepsilon}\left(u^{\varepsilon}\right) \geq F_{*}$, we obtain

$$
\int_{\Omega} k_{\varepsilon}(w) \leq \frac{1}{F_{*}^{p}}\left(\int_{\Omega} k_{\varepsilon}(w)\left|\nabla u^{\varepsilon}\right|^{p}+\int_{\Omega} F_{\varepsilon}^{p}\left(u^{\varepsilon}\right)\right) .
$$

As $F$ is continuous and $\left(u^{\varepsilon}\right)_{\varepsilon}$ is uniformly bounded in $L^{\infty}(\Omega),\left(F_{\varepsilon}^{p}\left(u^{\varepsilon}\right)\right)_{\varepsilon}$ is also uniformly bounded in $L^{\infty}(\Omega)$. Using (20) and (21) we obtain, from (19), that

$$
\left(a_{*}-a^{*}\left(\alpha_{\varepsilon}-\frac{\beta_{\varepsilon}}{F_{*}^{p}}\right)\right) \int_{\Omega} k_{\varepsilon}(w)\left|\nabla u^{\varepsilon}\right|^{p} \leq \frac{\beta_{\varepsilon}}{F_{*}^{p}}\left\|F_{\varepsilon}^{p}\left(u^{\varepsilon}\right)\right\|_{L^{\infty}(\Omega)}+\left\|f_{\varepsilon}\right\|_{L^{1}(\Omega)}\left\|u^{\varepsilon}\right\|_{L^{\infty}(\Omega)},
$$

The right hand side of the above inequality is bounded by a positive constant $C$ independent of $\varepsilon$. Choosing $\varepsilon$ sufficiently small such that $\alpha_{\varepsilon}-\frac{\beta_{\varepsilon}}{F_{*}^{p}} \leq \frac{a_{*}}{2 a^{*}}$ we get

$$
\int_{\Omega} k_{\varepsilon}(w)\left|\nabla u^{\varepsilon}\right|^{p} \leq \frac{2}{a_{*}} C
$$

and, using this inequality and (22), we immediately obtain (17). 
Denote $A_{\varepsilon}=\left\{x \in \Omega:\left|\nabla u^{\varepsilon}(x)\right|^{p}>F_{\varepsilon}^{p}\left(u^{\varepsilon}(x)\right)+\varepsilon\right\}$. Observe that, for $q>p$,

$$
\begin{aligned}
\int_{\Omega}\left|\nabla u^{\varepsilon}\right|^{q}=\int_{\Omega \backslash A_{\varepsilon}}\left|\nabla u^{\varepsilon}\right|^{q} & +\int_{A_{\varepsilon}}\left|\nabla u^{\varepsilon}\right|^{q} \\
& \leq|\Omega|\left\|F_{\varepsilon}^{p}+\varepsilon\right\|_{L^{\infty}(-M, M)}^{\frac{q}{p}}+2^{\frac{q}{p}-1}\left(\int_{A_{\varepsilon}}\left(\left|\nabla u^{\varepsilon}\right|^{p}-F_{\varepsilon}^{p}\left(u^{\varepsilon}\right)\right)^{\frac{q}{p}}+\int_{A_{\varepsilon}} F_{\varepsilon}^{q}\left(u^{\varepsilon}\right)\right)
\end{aligned}
$$

and to conclude the boundedness of $\left\|\nabla u^{\varepsilon}\right\|_{L^{q}(\Omega)}$, we only need to control the second term of the right hand side of the above inequality. As, for all $j \in \mathbb{N}$ and $s>0$ we have $e^{s} \geq s^{j} / j$ !, then, for $\frac{q}{p} \in \mathbb{N}$, we get, by the definition of $k_{\varepsilon}$,

$$
\int_{A_{\varepsilon}} w^{\frac{q}{p}} \leq \varepsilon^{\frac{q}{p}}\left(\frac{q}{p}\right) ! \int_{A_{\varepsilon}} k_{\varepsilon}(w)
$$

and, by (17), the conclusion follows, first for $q$ such that $\frac{q}{p} \in \mathbb{N}$ and after for any $1<q<\infty$.

Proof of Theorem 1.3 Let $u^{\varepsilon}$ be the solution of problem (14). From (16)-(18), we get that there exists $u \in W^{1, q}(\Omega)$ such that, at least for a subsequence,

$$
\begin{aligned}
& \nabla u^{\varepsilon} \underset{\varepsilon \rightarrow 0}{\longrightarrow} \nabla u \text { weakly in } L^{q}(\Omega), \text { for any } 1<q<\infty, \\
& u^{\varepsilon} \underset{\varepsilon \rightarrow 0}{\longrightarrow} u \text { in } \mathscr{C}(\bar{\Omega}) .
\end{aligned}
$$

Let us prove that $u \in \mathbb{K}_{F(u)}$. Set

$$
B_{\varepsilon}=\left\{x \in \Omega:\left|\nabla u^{\varepsilon}(x)\right|^{p}-F_{\varepsilon}^{p}\left(u^{\varepsilon}(x)\right) \geq \sqrt{\varepsilon}\right\} .
$$

Then, as $k_{\varepsilon}$ is nondecreasing, and using (17)

$$
\left|B_{\varepsilon}\right|=\int_{B_{\varepsilon}} 1 \leq \int_{B_{\varepsilon}} \frac{k_{\varepsilon}\left(\left|\nabla u^{\varepsilon}(x)\right|^{p}-F_{\varepsilon}^{p}\left(u^{\varepsilon}(x)\right)\right)}{k_{\varepsilon}(\sqrt{\varepsilon})} \leq C e^{-\frac{1}{\sqrt{\varepsilon}}} .
$$

Let $\omega$ be any measurable subset of $\Omega$. As

$$
\left|\nabla u^{\varepsilon}\right|^{p}-F_{\varepsilon}^{p}\left(u^{\varepsilon}\right)-\sqrt{\varepsilon} \underset{\varepsilon \rightarrow 0}{\longrightarrow}|\nabla u|^{p}-F^{p}(u) \text { weakly in } L^{1}(\Omega),
$$

then

$$
\begin{aligned}
\int_{\omega}\left(|\nabla u|^{p}-F^{p}(u)\right) & =\lim _{\varepsilon \rightarrow 0} \int_{\omega}\left(\left|\nabla u^{\varepsilon}\right|^{p}-F_{\varepsilon}^{p}\left(u^{\varepsilon}\right)-\sqrt{\varepsilon}\right) \\
& \leq \lim _{\varepsilon \rightarrow 0} \int_{\omega \cap B_{\varepsilon}}\left|\nabla u^{\varepsilon}\right|^{p} \\
& =\lim _{\varepsilon \rightarrow 0}\left|\omega \cap B_{\varepsilon}\right|^{\frac{1}{2}}\left\|\nabla u^{\varepsilon}\right\|_{L^{2 p}(\Omega)}^{p}=0, \quad \text { using (23) and (18), }
\end{aligned}
$$

concluding that $|\nabla u| \leq F(u)$ a.e. in $\Omega$.

Let us now see that $u$ solves the quasivariational inequality (8). Given $v \in \mathbb{K}_{F(u)}$ we define $\gamma_{\varepsilon}=$ $\left\|F(u)-F_{\varepsilon}\left(u^{\varepsilon}\right)\right\|_{\mathscr{C}(\bar{\Omega})}$ and $v^{\varepsilon}=\frac{F_{*}}{F_{*}+\gamma_{\varepsilon}} v$. Observe that $v^{\varepsilon} \in \mathbb{K}_{F_{\varepsilon}\left(u^{\varepsilon}\right)}$ and $v^{\varepsilon} \underset{\varepsilon \rightarrow 0}{\longrightarrow} v$ in $W^{1, p}(\Omega)$. Besides, denoting again $w=\left|\nabla u^{\varepsilon}\right|^{p}-F_{\varepsilon}^{p}\left(u^{\varepsilon}\right)$,

$$
\begin{aligned}
\left(k_{\varepsilon}(w)-1\right) a_{\varepsilon}(x)\left(\left|\nabla u^{\varepsilon}\right|^{2}+\varepsilon\right)^{\frac{p-2}{2}} & \nabla u^{\varepsilon} \cdot \nabla\left(v^{\varepsilon}-u^{\varepsilon}\right) \\
& \leq\left(k_{\varepsilon}(w)-1\right) a_{\varepsilon}(x)\left(\left|\nabla u^{\varepsilon}\right|^{2}+\varepsilon\right)^{\frac{p-2}{2}}\left|\nabla u^{\varepsilon}\right|\left(\left|\nabla v^{\varepsilon}\right|-\left|\nabla u^{\varepsilon}\right|\right) \leq 0,
\end{aligned}
$$

as, when $k_{\varepsilon}(w)>1$ then $\left|\nabla u^{\varepsilon}\right| \geq F_{\varepsilon}\left(u^{\varepsilon}\right) \geq\left|\nabla v^{\varepsilon}\right|$.

So, multiplying equation (14a) by $v^{\varepsilon}-u^{\varepsilon}$, and using (24), we obtain

$$
\begin{aligned}
\int_{\Omega} a_{\varepsilon}(x)\left(\left|\nabla u^{\varepsilon}\right|^{2}+\varepsilon\right)^{\frac{p-2}{2}} \nabla u^{\varepsilon} \cdot \nabla\left(v^{\varepsilon}-u^{\varepsilon}\right)+\varepsilon \int_{\Omega} \nabla u^{\varepsilon} \cdot \nabla\left(v^{\varepsilon}-u^{\varepsilon}\right) & +\int c\left|u^{\varepsilon}\right|^{p-2} u^{\varepsilon}\left(v^{\varepsilon}-u^{\varepsilon}\right) \geq \int_{\Omega} f^{\varepsilon}\left(v^{\varepsilon}-u^{\varepsilon}\right) .
\end{aligned}
$$


Using the strict monotonicity of the $p$-laplacian operator, we get

$$
\begin{aligned}
\int_{\Omega} a_{\varepsilon}(x)\left(\left|\nabla v^{\varepsilon}\right|^{2}+\varepsilon\right)^{\frac{p-2}{2}} \nabla v^{\varepsilon} \cdot \nabla\left(v^{\varepsilon}-u^{\varepsilon}\right)+\varepsilon \int_{\Omega} \nabla u^{\varepsilon} \cdot \nabla\left(v^{\varepsilon}-u^{\varepsilon}\right) & +\int c\left|u^{\varepsilon}\right|^{p-2} u^{\varepsilon}\left(v^{\varepsilon}-u^{\varepsilon}\right) \geq \int_{\Omega} f^{\varepsilon}\left(v^{\varepsilon}-u^{\varepsilon}\right)
\end{aligned}
$$

and, letting $\varepsilon \rightarrow 0$ and, as the term $\int_{\Omega} \nabla u^{\varepsilon} \cdot \nabla\left(v^{\varepsilon}-u^{\varepsilon}\right)$ is bounded, we have

$$
\int_{\Omega} a(x)|\nabla v|^{p-2} \nabla v \cdot \nabla(v-u)+\int c|u|^{p-2} u(v-u) \geq \int_{\Omega} f(v-u),
$$

which implies, by applying a kind of Minty's Lemma, that

$$
\int_{\Omega} a(x)|\nabla u|^{p-2} \nabla u \cdot \nabla(v-u)+\int c|u|^{p-2} u(v-u) \geq \int_{\Omega} f(v-u)
$$

which concludes the proof of the theorem.

\section{The case with Dirichlet boundary condition}

In this section we consider the quasivariational case with nonhomogeneous Dirichlet boundary condition, correspondent to the convex sets defined in (3), with $\varphi$ substituted by $F(u)$. As it was already referred, one main concern is to avoid the emptiness of these sets. So we introduce the assumption (10), based on a compatibility condition between the boundary condition $g$, the minimum of the gradient constraint function $F$ and the geometry of the domain.

Consider the variational inequality: to find $u \in \mathcal{K}_{\varphi}$ such that

$$
\int_{\Omega} \boldsymbol{a}(x, \nabla u) \cdot \nabla(v-u)+\int_{\Omega} c|u|^{p-2} u(v-u) \geq \int_{\Omega} f(v-u), \quad \forall v \in \mathcal{K}_{\varphi},
$$

where $\mathcal{K}_{\varphi}$ is defined in (3).

Proof of Theorem 1.4 The proof follows the steps of the proof of Theorem 1.1. The main difference consists in proving the continuity of the operator $T: \mathscr{C}(\bar{\Omega}) \longrightarrow W^{1, p}(\Omega)$, where $T(\varphi)$ is the solution of problem (25), with $F(\varphi)$ in the place of $\varphi$. We will sketch the proof of the Mosco convergence of $\mathcal{K}_{F\left(\varphi_{n}\right)}$ to $\mathcal{K}_{F(\varphi)}$, where $\left(\varphi_{n}\right)_{n}$ converges to $\varphi$ in $\mathscr{C}(\bar{\Omega})$, from which we immediately deduce the continuity of $T$ (see [12] or [16, Theorem 4.1]). So, we only need to prove the following two conditions:

$$
\forall v \in \mathcal{K}_{F(\varphi)} \forall n \in \mathbb{N} \exists v_{n} \in \mathcal{K}_{F\left(\varphi_{n}\right)}: \quad v_{n} \underset{n}{\longrightarrow} v \text { in } W^{1, p}(\Omega),
$$

$$
\text { if, for all } n \in \mathbb{N}, v_{n} \in \mathcal{K}_{F\left(\varphi_{n}\right)} \text { and } v_{n} \underset{n}{\longrightarrow} v \text { in } W^{1, p}(\Omega) \text {, then } v \in \mathcal{K}_{F(\varphi)} \text {. }
$$

Using the assumption (10) we may extend the function $g$ to $\bar{\Omega}$ (still calling it by $g$ ) satisfying the condition $|\nabla g|=k F_{*}$ (see [9]).

To prove (26a) consider, for given $v \in \mathcal{K}_{F(\varphi)}$ and, for $n \in \mathbb{N}$,

$$
G_{n}=\min \left\{F\left(\varphi_{n}\right), F(\varphi)\right\}
$$

and $v_{n}=b_{n} v+\left(1-b_{n}\right) g$, where

$$
b_{n}=\min _{x \in \bar{\Omega}} \frac{G_{n}(x)-k F_{*}}{F(\varphi(x))-k F_{*}} .
$$

So, $0<b_{n} \leq 1$ and $\left(\frac{G_{n}-k F_{*}}{F(\varphi)-k F_{*}}\right)_{n}$ converges to 1 in $\mathscr{C}(\bar{\Omega})$ and, as $\bar{\Omega}$ is compact, $b_{n} \underset{n}{\longrightarrow} 1$. Note that $v_{n} \in \mathcal{K}_{F\left(\varphi_{n}\right)}$ as $v_{\left.n\right|_{\partial \Omega}}=g$ and

$$
\left|\nabla v_{n}(x)\right| \leq b_{n} F(\varphi(x))+\left(1-b_{n}\right) k F_{*} \leq G_{n}(x),
$$


because $b_{n} \leq \frac{G_{n}(x)-k F_{*}}{F(\varphi(x))-k F_{*}}$. We have

$$
\int_{\Omega}\left|\nabla\left(v_{n}-v\right)\right|^{p}=\left(1-b_{n}\right)^{p} \int_{\Omega}|\nabla(g-v)|^{p} \underset{n}{\longrightarrow} 0 .
$$

To prove (26b), let $\left(v_{n}\right)_{n}$ be a sequence in $\mathcal{K}_{F\left(\varphi_{n}\right)}$, converging weakly in $W^{1, p}(\Omega)$ to $v$. As $v_{\left.n\right|_{\partial \Omega}}=g$ then $v_{\mid \partial \Omega}=g$. Given any measurable set $\omega \subset \Omega$,

$$
\int_{\omega}|\nabla v| \leq \liminf _{n} \int_{\omega}\left|\nabla v_{n}\right| \leq \liminf _{n} \int_{\omega} F\left(\varphi_{n}\right)=\int_{\omega} F(\varphi),
$$

so $|\nabla v| \leq F(\varphi)$ a.e. in $\Omega$, which means $v \in \mathcal{K}_{F(\varphi)}$. This concludes the proof of the continuity of $T$.

We present now an a priori estimate for the $W^{1, p}(\Omega)$ norm of $u_{\varphi}=T(\varphi)$, independent of $\varphi$.

Choosing $g$ as test function in (25) and recalling that $f \in L^{q^{\prime}}(\Omega)$ we have

$$
\begin{aligned}
& \int_{\Omega} \boldsymbol{a}\left(x, \nabla u_{\varphi}\right) \cdot \nabla u_{\varphi}+\int_{\Omega} c\left|u_{\varphi}\right|^{p} \leq \int_{\Omega} \boldsymbol{a}\left(x, \nabla u_{\varphi}\right) \cdot \nabla g+\int_{\Omega} f u_{\varphi}-\int_{\Omega} f g+\int_{\Omega} c\left|u_{\varphi}\right|^{p-2} u_{\varphi} g \\
& \leq \int_{\Omega}\left|\nabla u_{\varphi}\right|^{p-1} a^{*} k F_{*}+\int_{\Omega}\left|u_{\varphi}\right|^{p-1}\|c g\|_{L^{\infty}(\Omega)}+\|f\|_{L^{q^{\prime}}(\Omega)}\left\|u_{\varphi}\right\|_{L^{q}(\Omega)}+\|f\|_{L^{q^{\prime}}(\Omega)}\|g\|_{L^{q}(\Omega)} .
\end{aligned}
$$

As $\left\|u_{\varphi}\right\|_{L^{q}(\Omega)} \leq C_{q}\left\|u_{\varphi}\right\|_{W^{1, p}(\Omega)}$ we have, for $\delta>0$,

$$
\begin{aligned}
a_{*}\left\|\nabla u_{\varphi}\right\|_{L^{p}(\Omega)}^{p} \leq \frac{\delta^{p^{\prime}}}{p^{\prime}}\left\|u_{\varphi}\right\|_{W^{1, p}(\Omega)}^{p}+\frac{|\Omega|}{\delta^{p} p}\left(\left(a^{*} k F_{*}\right)^{p}+\|c g\|_{L^{\infty}(\Omega)}^{p}\right) & \\
& +\frac{\delta^{p}}{p}\left\|u_{\varphi}\right\|_{W^{1, p}(\Omega)}^{p}+\frac{C_{q}^{p^{\prime}}}{\delta p^{\prime} p^{\prime}}\|f\|_{L^{q^{\prime}}(\Omega)}^{p^{\prime}}+\|f\|_{L^{q^{\prime}}(\Omega)}\|g\|_{L^{q}(\Omega)} .
\end{aligned}
$$

Applying the Poincaré inequality to $u_{\varphi}$, we have

$$
\left\|u_{\varphi}\right\|_{W^{1, p}(\Omega)}^{p} \leq c_{p}\left(\left\|\nabla u_{\varphi}\right\|_{L^{p}(\Omega)}^{p}+\|g\|_{L^{p}(\partial \Omega)}^{p}\right) .
$$

Choosing $\delta$ such that $\left(\frac{\delta^{p^{\prime}}}{p^{\prime}}+\frac{\delta^{p}}{p}\right) c_{p}<a_{*}$, we conclude that there exists a positive constant $C$, depending on $\|f\|_{L^{q^{\prime}}(\Omega)}$ and $\|g\|_{L^{\infty}(\Omega)}$, such that $\left\|\nabla u_{\varphi}\right\|_{L^{p}(\Omega)}^{p} \leq C$. Applying again the Poincaré inequality, there exists another positive constant $C$ such that

$$
\left\|u_{\varphi}\right\|_{W^{1, p}(\Omega)}^{p} \leq C .
$$

As we proved the continuity of the operator $T$ and the above estimate, the conclusion follows as in the proof of Theorem 1.1.

The proof of Theorem 1.5 will be done using, as in the proof of Theorem 1.3, a family of approximating problems, obtained by regularizing and penalizing the quasivariational inequality. For $0<\varepsilon<1$, consider the approximating family of problems:

$$
\begin{gathered}
-\nabla \cdot\left(k_{\varepsilon}\left(\left|\nabla u^{\varepsilon}\right|^{p}-F_{\varepsilon}^{p}\left(u^{\varepsilon}\right)\right) a_{\varepsilon}(x)\left(\left|\nabla u^{\varepsilon}\right|^{2}+\varepsilon\right)^{\frac{p-2}{2}} \nabla u^{\varepsilon}+\varepsilon \nabla u^{\varepsilon}\right)+c\left|u^{\varepsilon}\right|^{p-2} u^{\varepsilon}=f^{\varepsilon} \quad \text { in } \Omega, \\
u_{\mid \partial \Omega}^{\varepsilon}=g^{\varepsilon},
\end{gathered}
$$

where $a_{\varepsilon}, g^{\varepsilon}, f^{\varepsilon}$ and $F_{\varepsilon}$ are approximations by convolution of $a, g, f$ and $F$, and $k_{\varepsilon}: \mathbb{R} \rightarrow \mathbb{R}$ is a smooth nondecreasing function as in (15).

This problem has a unique solution $u^{\varepsilon} \in \mathscr{C}^{2, \alpha}(\Omega) \cap \mathscr{C}(\bar{\Omega})$ (see [5, Theorem 5.19]).

Consider an extension $g^{\varepsilon}$ to $\bar{\Omega}$, still denoted by $g^{\varepsilon}$, such that $\left|\nabla g^{\varepsilon}\right|=k F_{*}$ in $\Omega$. Notice that such a function exists because (10) is verified and $g^{\varepsilon} \in W^{1, \infty}(\Omega)$.

Proposition 3.1 Under the assumptions of Theorem 1.5 there exist positive constants $C$ and $D_{q}$, independent of $\varepsilon$, such that

$$
\begin{gathered}
\left\|u^{\varepsilon}\right\|_{L^{\infty}(\Omega)} \leq C, \\
\left\|k_{\varepsilon}\left(\left|\nabla u^{\varepsilon}\right|^{p}-F_{\varepsilon}^{p}\left(u^{\varepsilon}\right)\right)\right\|_{L^{1}(\Omega)} \leq C, \\
\forall 1<q<\infty \quad\left\|\nabla u_{\varepsilon}\right\|_{L^{q}(\Omega)} \leq D_{q} .
\end{gathered}
$$


Proof By the strong maximum principle for quasilinear elliptic equations, the $L^{\infty}(\Omega)$-norm of $u^{\varepsilon}$ depends only on $\|f\|_{L^{\infty}(\Omega)}$ and $\|g\|_{L^{\infty}(\partial \Omega)}$.

Denote once again $\left|\nabla u^{\varepsilon}\right|^{p}-F_{\varepsilon}^{p}\left(u^{\varepsilon}\right)$ by $w$. Multiplying by $u^{\varepsilon}-g^{\varepsilon}$ the equation (27a) and integrating over $\Omega$ we obtain

$$
\begin{aligned}
\int_{\Omega} & k_{\varepsilon}(w) a_{\varepsilon}(x)\left(\left|\nabla u^{\varepsilon}\right|^{2}+\varepsilon\right)^{\frac{p-2}{2}}\left|\nabla u^{\varepsilon}\right|^{2}+\varepsilon \int_{\Omega}\left|\nabla u^{\varepsilon}\right|^{2}+\int_{\Omega} c\left|u^{\varepsilon}\right|^{p} \\
= & \int_{\Omega} k_{\varepsilon}(w) a_{\varepsilon}(x)\left(\left|\nabla u^{\varepsilon}\right|^{2}+\varepsilon\right)^{\frac{p-2}{2}} \nabla u^{\varepsilon} \cdot \nabla g^{\varepsilon}+\varepsilon \int_{\Omega} \nabla u^{\varepsilon} \cdot \nabla g^{\varepsilon}+\int_{\Omega} c\left|u^{\varepsilon}\right|^{p-2} u^{\varepsilon} g^{\varepsilon}+\int_{\Omega} f^{\varepsilon}\left(u^{\varepsilon}-g^{\varepsilon}\right) .
\end{aligned}
$$

We can rewrite the above equality as

$$
\begin{aligned}
\int_{\Omega} k_{\varepsilon}(w) a_{\varepsilon}(x)\left(\left|\nabla u^{\varepsilon}\right|^{2}+\varepsilon\right)^{\frac{p}{2}}+\varepsilon \int_{\Omega}\left|\nabla u^{\varepsilon}\right|^{2} & +\int_{\Omega} c\left|u^{\varepsilon}\right|^{p} \\
=\varepsilon \int_{\Omega} k_{\varepsilon}(w) a_{\varepsilon}(x)\left(\left|\nabla u^{\varepsilon}\right|^{2}+\varepsilon\right)^{\frac{p-2}{2}} & +\int_{\Omega} k_{\varepsilon}(w) a_{\varepsilon}(x)\left(\left|\nabla u^{\varepsilon}\right|^{2}+\varepsilon\right)^{\frac{p-2}{2}} \nabla u^{\varepsilon} \cdot \nabla g^{\varepsilon} \\
& \quad+\varepsilon \int_{\Omega} \nabla u^{\varepsilon} \cdot \nabla g^{\varepsilon}+\int_{\Omega} c\left|u^{\varepsilon}\right|^{p-2} u^{\varepsilon} g^{\varepsilon}+\int_{\Omega} f^{\varepsilon}\left(u^{\varepsilon}-g^{\varepsilon}\right)
\end{aligned}
$$

and then, using (28) and (30), there exists a constant $C_{1}>0$, independent of $\varepsilon$, such that

$$
a_{*} \int_{\Omega} k_{\varepsilon}(w)\left(\left|\nabla u^{\varepsilon}\right|^{2}+\varepsilon\right)^{\frac{p}{2}} \leq a^{*} \varepsilon \int_{\Omega} k_{\varepsilon}(w)\left(\left|\nabla u^{\varepsilon}\right|^{2}+\varepsilon\right)^{\frac{p-2}{2}}+a^{*} k F_{*} \int_{\Omega} k_{\varepsilon}(w)\left(\left|\nabla u^{\varepsilon}\right|^{2}+\varepsilon\right)^{\frac{p-2}{2}}\left|\nabla u^{\varepsilon}\right|+C_{1} \text {. }
$$

Observe that

$$
\begin{aligned}
a^{*} k F_{*} \int_{\Omega} k_{\varepsilon}(w)\left(\left|\nabla u^{\varepsilon}\right|^{2}+\varepsilon\right)^{\frac{p-2}{2}}\left|\nabla u^{\varepsilon}\right| \leq a^{*} k F_{*} \int_{\Omega} k_{\varepsilon}(w)\left(\left|\nabla u^{\varepsilon}\right|^{2}+\varepsilon\right)^{\frac{p-1}{2}} & \leq a^{*} k F_{*} \int_{\Omega} k_{\varepsilon}(w)\left(\frac{\left.\left|\nabla u^{\varepsilon}\right|^{2}+\varepsilon\right)^{\frac{p}{2}}}{p^{\prime} \delta^{p^{\prime}}}+\frac{\delta^{p}}{p}\right)
\end{aligned}
$$

for any $\delta>0$. Choosing $\delta=F_{*}^{\frac{1}{p^{\prime}}}$, we obtain

$$
a^{*} k F_{*} \int_{\Omega} k_{\varepsilon}(w)\left(\left|\nabla u^{\varepsilon}\right|^{2}+\varepsilon\right)^{\frac{p-2}{2}}\left|\nabla u^{\varepsilon}\right| \leq \frac{a^{*} k}{p^{\prime}} \int_{\Omega} k_{\varepsilon}(w)\left(\left|\nabla u^{\varepsilon}\right|^{2}+\varepsilon\right)^{\frac{p}{2}}+\frac{a^{*} k F_{*}^{p}}{p} \int_{\Omega} k_{\varepsilon}(w) .
$$

Inequalities (31) and (21) allow us to obtain

$$
\left(a_{*}-\frac{a^{*} k}{p^{\prime}}-\alpha_{\varepsilon}\right) \int_{\Omega} k_{\varepsilon}(w)\left|\nabla u^{\varepsilon}\right|^{p} \leq\left(\frac{a^{*} k F_{*}^{p}}{p}+\beta_{\varepsilon}\right) \int_{\Omega} k_{\varepsilon}(w)+C_{1} .
$$

Recalling (22), we obtain

$$
\left(F_{*}^{p}\left(a_{*}-a^{*} k\right)-\left(F_{*}^{p} \alpha_{\varepsilon}+\beta_{\varepsilon}\right)\right) \int_{\Omega} k_{\varepsilon}(w) \leq\left(a_{*}-\frac{a^{*} k}{p^{\prime}}-\alpha_{\varepsilon}\right) \int_{\Omega} F_{\varepsilon}^{p}\left(u^{\varepsilon}\right)+C_{1} .
$$

Observing, as in the proof of Proposition 2.4, that $\left(F_{\varepsilon}^{p}\left(u^{\varepsilon}\right)\right)_{\varepsilon}$ is uniformly bounded in $L^{\infty}(\Omega)$ and $F_{*}^{p} \alpha_{\varepsilon}+$ $\beta_{\varepsilon} \underset{\varepsilon \rightarrow 0}{\longrightarrow} 0$, there exists $D>0$ such that

$$
F_{*}^{p} \frac{a_{*}-a^{*} k}{2} \int_{\Omega} k_{\varepsilon}(w) \leq D
$$

and the conclusion (29) follows.

The proof of (30) is similar to the case of Neumann boundary condition.

Remark 3.2 If $a_{*}=a^{*}$, as in the $p$-laplacian case, the only restriction on $k$ is $0<k<1$.

Proof of Theorem 1.5 After the previous proposition, the proof of this theorem is similar to the proof of Theorem 1.3. Here the constant $c_{*}$ may be zero. However, after obtaining the uniform control of $\left\|\nabla u^{\varepsilon}\right\|_{L^{q}(\Omega)}$, the Poincaré inequality implies the uniform boundedness of $\left\|u^{\varepsilon}\right\|_{W^{1, q}(\Omega)}$. The verification that $u_{\left.\right|_{\partial \Omega}}=g$ is a consequence of the equality $u_{\mid \partial \Omega}^{\varepsilon}=g^{\varepsilon}$ and convergence of $\left(u^{\varepsilon}\right)_{\varepsilon}$ to $u$ in $\mathscr{C}(\bar{\Omega})$. 


\section{Acknowledgments}

The authors are grateful to the referees for valuable comments and suggestions that helped to improve the text.

This research was partially supported by CMAT - "Centro de Matemática da Universidade do Minho", financed by FEDER Funds through "Programa Operacional Factores de Competitividade - COMPETE" and by Portuguese Funds through FCT, "Fundação para a Ciência e a Tecnologia", within the Project EstC/MAT/UI0013/ 2011.

\section{References}

[1] A. Azevedo, F. Miranda and L. Santos, Variational and quasivariational inequalities with first order constraints, J. Math. Anal. Appl. 397 (2013) 738-756.

[2] J. W. Barrett and L. Prigozhin, A quasi-variational inequality problem in superconductivity, Math. Models Methods Appl. Sci. 20(5) (2010) 679-706.

[3] H. Brezis, L. A. Caffarelli and A. Friedman, Reinforcement problems for elliptic equations and variational inequalities, Ann. Mat. Pura Appl. (4) 123 (1980) 219-246.

[4] L. A. Caffarelli and A. Friedman, Reinforcement problems in elastoplasticity, Rocky Mountain J. Math. 10(1) (1980) 155-184.

[5] D. Gilbarg and N. S. Trudinger, Elliptic partial differential equations of second order, Classics in Mathematics, Springer-Verlag, Berlin, 2001.

[6] D. Kinderlehrer and G. Stampacchia, An introduction to variational inequalities and their applications, Volume 88 of Pure and Applied Mathematics, Academic Press Inc., New York, 1980.

[7] M. Kunze and J. F. Rodrigues, An elliptic quasi-variational inequality with gradient constraints and some of its applications, Math. Methods Appl. Sci. 23(10) (2000) 897-908.

[8] J. L. Lions, Quelques méthodes de résolution des problèmes aux limites non linéaires, Dunod, Paris 1969.

[9] P. L. Lions, Generalized solutions of Hamilton-Jacobi equations, Volume 69 of Research Notes in Mathematics, Pitman, Boston, 1982.

[10] F. Miranda, J. F. Rodrigues and L. Santos. A class of stationary nonlinear Maxwell systems, Math. Models Methods Appl. Sci. 19(10) (2009) 1883-1905.

[11] F. Miranda, J. F. Rodrigues and L. Santos, On a p-curl system arising in electromagnetism, Discrete Contin. Dyn. Syst. Ser. S 5(3) (2012) 605-629.

[12] U. Mosco, Convergence of convex sets and of solutions of variational inequalities, Advances in Math. 3 (1969) 510-585.

[13] L. Prigozhin, Sandpiles and river networks: extended systems with nonlocal interactions, Phys. Rev. E (3) 49(2) (1994) 1161-1167.

[14] L. Prigozhin, On the Bean critical-state model in superconductivity, European J. Appl. Math. 7(3) (1996) 237-247.

[15] L. Prigozhin, Variational model of sandpile growth, European J. Appl. Math. 7(3) (1996) 225-235.

[16] J. F. Rodrigues, Obstacle problems in mathematical physics. North-Holland, Amsterdam, (1987).

[17] J. F. Rodrigues and L. Santos, A parabolic quasi-variational inequality arising in a superconductivity model, Ann. Scuola Norm. Sup. Pisa Cl. Sci. (4) 29(1) (2000) 153-169.

[18] T. W. Ting, Elastic-plastic torsion problem II, Arch. Rational Mech. Anal. 25 (1967) 342-366. 